\title{
Combining wearable finger haptics and Augmented Reality: User evaluation using an external camera and the Microsoft HoloLens
}

\author{
Leonardo Meli ${ }^{1}$, Claudio Pacchierotti ${ }^{2}$, Gionata Salvietti ${ }^{1,3}$, Francesco Chinello ${ }^{4}$, \\ Maurizio Maisto ${ }^{5}$, Alessandro De Luca ${ }^{5}$, Domenico Prattichizzo ${ }^{1,3}$
}

\begin{abstract}
Augmented Reality (AR) enriches our physical world with digital content and media, such as 3D models and videos, overlaying in real time the camera view of our smartphone, tablet, laptop, or glasses. Despite the recent massive interest for this technology, it is still not possible to receive rich haptic feedback when interacting with augmented environments. This lack is mainly due to the poor diffusion of suitable haptic interfaces, which should be easy to wear, lightweight, compact, and inexpensive. In this paper, we briefly review the state of the art on wearable haptics and its application in AR. Then, we present three AR use cases, considering tasks of manipulation, guidance, and gaming, using both external cameras with standard screens as well as fully-wearable solutions, using the Microsoft HoloLens. We evaluate these tasks enrolling a total of 34 subjects, analyzing performance and user experience when using a 3-DoF wearable device for the fingertip, a 2-DoF wearable device for the proximal finger phalanx, a vibrotactile ring, and a popular sensory substitution technique (interaction force displayed as a colored bar). Results show that providing haptic feedback through the wearable devices significantly improves the performance, intuitiveness, and comfort of the considered AR tasks.
\end{abstract}

Index Terms-Haptics and Haptic Interfaces, Wearable Robots, Human-Centered Robotics.

\section{INTRODUCTION}

$\mathbf{C}$ OMPACT, unobtrusive, easy-to-wear, and lightweight haptic devices enable researchers to provide compelling touch sensations, significantly increasing the immersiveness and performance of VR and AR environments. Recent technologies to provide informative and compelling unobtrusive haptic stimuli include moving platforms, pin-arrays, shearing belts and tactors, pneumatic jets, and balloon-based systems [1]. Frisoli

Manuscript received: February, 23, 2018; Revised May, 2, 2018; Accepted July, 13, 2018.

This paper was recommended for publication by Editor Yasuyoshi Yokokohji upon evaluation of the Associate Editor and Reviewers' comments. The research leading to these results has received funding from the European Union's Horizon 2020 Research and Innovation Programme under Grant Agreement $\mathrm{n}^{\circ} 688857$ of the project "SoftPro".

${ }^{1}$ Meli, Salvietti, and Prattichizzo are with Dept. Information Engineering and Mathematics, Univ. of Siena, Italy. \{meli, salviettigio, prattichizzo\}@diism.unisi.it

${ }^{2}$ Pacchierotti is with CNRS, Univ Rennes, Inria, IRISA, Rennes, France. claudio.pacchierotti@irisa.fr

${ }^{3}$ Salvietti and Prattichizzo are also with Dept. Advanced Robotics, Istituto Italiano di Tecnologia, Italy.

${ }^{4}$ Chinello is with Dept. Businesses Development and Technology, Aarhus Univ., Denmark. chinello@btech.au.dk

5 Maisto and De Luca are with Dip. Ingegneria Informatica, Automatica e Gestionale, Sapienza Universita di Roma, Italy. \{maisto, deluca\}@diag.uniroma1.it

Digital Object Identifier (DOI): see top of this page.

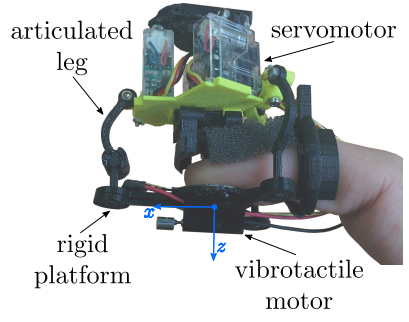

(a) Chinello et al. [5], [6]

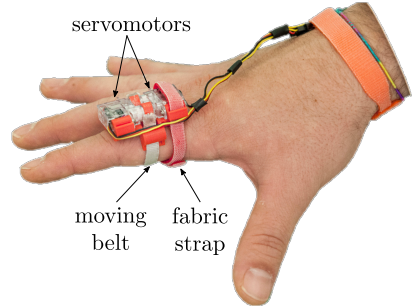

(b) Pacchierotti et al. [10]
Fig. 1. Two of the wearable haptic devices employed in our study.

et al. [2] presented a device composed of a parallel platform and a serial wrist; the parallel platform actuates a translation stage for positioning the plate with respect to the fingerpad, while the wrist is in charge of adjusting its orientation. Gabardi et al. [3] further improved this device by replacing sheathed tendon actuation with DC motors mounted directly on the joints. Prattichizzo et al. [4] developed a wearable 3-DoF fingertip device consisting of two platforms: one located on the nail side of the finger, housing three DC motors, and the other one located in contact with the finger pulp. The two platforms are connected by three cables. A new version of this device featuring three articulated rigid legs connecting the two platforms has been presented in [5], [6]. More recently, Leonardis et al. [7] developed a 3RSR wearable skin stretch device for the fingertip. It moves a rigid tactor in contact with the skin, providing skin stretch and making/breaking contact sensations. Girard et al. [8] developed a wearable fingertip device capable of rendering 2-DoF skin stretch stimuli. Two DC motors move a tactor in contact with the finger pulp, achieving a maximum displacement of $2 \mathrm{~mm}$ in both directions. Schorr and Okamura [9] presented a wearable device composed of a delta parallel mechanism, capable of making/breaking contact with the fingertip, as well as rendering shear and normal skin deformation stimuli. The device has three translational DoF, enabling normal, lateral, and longitudinal skin deformation.

While most of the above wearable haptic devices have been evaluated and tested in VR, the use of wearable haptics in AR is still at a very early stage. This is mainly due to the fact that many wearable devices are designed for the fingertip [1], impairing the users when they need to interact with real objects. For this reason, few haptic feedback systems have been used in AR so far. For example, Scheggi et al. [11] used a grounded haptic interface and two wearable fingertip devices to provide, at the same time, haptic information about the 
shape and the weight of a virtual object superimposed on a real object. More recently, Maisto et al. [12] presented the experimental evaluation of two wearable haptic interfaces for the fingers in AR. The first one is a 3-DoF fingertip device, which is similar to that presented in [4] and applies tactile stimuli through a moving platform; the second one is a $2-\mathrm{DoF}$ skin stretch device for the finger and applies tactile stimuli through a soft belt. De Tinguy et al. [13] used a similar 2-DoF skin stretch device to alter the perceived stiffness of tangible objects in AR. Other haptic technologies used in AR include electrostimulation [14], haptic vibratory belts [15], [16], and string-based haptic device [17].

In this paper, we evaluate the role that wearable finger haptics may play in augmented reality. We consider different tasks involving manipulation, guidance, and gaming, using three wearable haptic interfaces providing haptic stimuli either at the fingertip or at the finger proximal phalanx. With respect to [12], in this work we evaluate the role of wearable haptics in a broader set of tasks and AR settings. Enrolling 34 different subjects in three different experiments, this work presents one the most extensive evaluations of wearable haptics in AR applications.

The main contributions of our work are:

- design of new modular settings and use cases in Augmented Reality, using either standard cameras and displays or fully-wearable solutions (i.e., MS HoloLens);

- design of a wireless wearable vibrotactile ring for the proximal phalanx;

- design of three user studies assessing the effectiveness of wearable haptics in the different AR contexts, considering for the first time tasks involving manipulation, guidance, and feedback for mid-air gestures;

- comparison, through statistical analysis, of the performance of a wearable device for the fingertip, a wearable devices for the proximal finger phalanx, and a sensory substitution technique vs. not providing any kind of haptic feedback.

\section{HAPTIC FEEDBACK METHODS}

We evaluated our AR scenarios using a wearable device for the fingertip (see Sec. II-A), a wearable device for the proximal finger phalanx (see Sec. II-B), a wearable vibrotactile ring (see Sec. II-C), and a sensory substitution technique (see Sec. II-D), comparing them with conditions providing no haptic feedback information. The characteristics of these devices are summarized in Tab. I.

\section{A. 3-DoF wearable device for the fingertip}

This device has been preliminary presented by Chinello et al. [5], [6] and it is shown in Fig. 1a. It consists of two sides: a static upper body located on the nail side of the finger and a mobile end-effector in contact with the finger pulp. The upper body part supports three light motors, while a FSR 402 Short sensor (Interlink Electronics, USA) is placed on the platform to detect contact with the finger pulp. The two parts are connected by three articulated legs, actuated by the motors. The end-effector can tilt and apply forces on the user's fingertip so to simulate a contacts with arbitrarily-oriented surfaces. A
TABLE I

SPECIFICATIONS FOR THE PROPOSED 3-DOF AND 2-DOF DEVICES.

\begin{tabular}{|c|c|c|c|c|}
\hline \multicolumn{2}{|c|}{ 3-DoF RRS device (Sec. II-A) } & \multicolumn{3}{|c|}{ 2-DoF device "hRing" (Sec. II-B) } \\
\hline Dimension & $35 \times 50 \times 48 \mathrm{~mm}$ & Dimens & & $31 \times 28 \times 12 \mathrm{~mm}$ \\
\hline Weight & $38 \mathrm{~g}$ & Weight & & $15 \mathrm{~g}$ \\
\hline Max. vertical displ. & $15 \mathrm{~mm}$ & Max. ve & al displ. & $13 \mathrm{~mm}$ \\
\hline Max. normal force & $4.7 \mathrm{~N}$ & Max. n & al force & $1.4 \mathrm{~N}$ \\
\hline Maximum Pitch & $\pi / 6 \mathrm{rad}$ & Max. hs & ontal displ. & $13 \mathrm{~mm}$ \\
\hline Maximum Roll & $\pi / 5 \mathrm{rad}$ & Pulleys & & $5 \mathrm{~mm}$ \\
\hline \multicolumn{5}{|c|}{ Vibrotactile ring (Sec. II-C) } \\
\hline \multicolumn{2}{|c|}{ Dimension } & & \multicolumn{2}{|c|}{$22 \times 24 \times 20 \mathrm{~mm}$} \\
\hline \multicolumn{2}{|l|}{ Weight } & & \multicolumn{2}{|l|}{$11 \mathrm{~g}$} \\
\hline \multicolumn{3}{|c|}{ Actuator vibrations frequency range } & \multicolumn{2}{|c|}{$110-240 \mathrm{~Hz}$} \\
\hline \multicolumn{3}{|c|}{ Actuator vibrations amplitude range } & \multicolumn{2}{|c|}{$0.5-1.7 \mathrm{G}$} \\
\hline
\end{tabular}

RRS (Revolute-Revolute-Spherical) kinematic chain constitutes the legs connecting the end-effector and to the upper body part. The wearable device weighs $38 \mathrm{~g}$ for $35 \times 50 \times 48 \mathrm{~mm}$ dimensions.

Fingertip deformation and applied wrench can be related by a non-linear impedance model, that depends on the fingertip specific features (e.g., geometry of the contact surface, subject's age). In this work, we assume a simplified linear fingertip impedance model representing the relationship between the resultant wrench and the platform displacement. Thus, we consider the platform configuration $\Delta \xi$ proportional to the wrench $-\mathbf{w}_{p}, \mathbf{w}_{p}=\mathbf{K} \Delta \boldsymbol{\xi}$, where $\mathbf{K} \in \mathbb{R}^{6 \times 6}$ is the fingertip stiffness matrix, as defined in [6]. From a desired wrench $\mathbf{w}_{p, d}$, we can therefore compute the corresponding desired platform configuration $\boldsymbol{\xi}_{d}=\boldsymbol{\xi}_{0}+\mathbf{K}^{-1} \mathbf{w}_{p, d}$ which is then obtained controlling the positions of the servo motors.

\section{B. 2-DoF wearable device for the finger, "hRing"}

This device has been preliminary presented by Pacchierotti et al. [10] and it is shown in Fig. 1b. It is composed of an ABS support that houses two servo motors and two pulleys, and a fabric belt that can apply normal and shear forces on the finger pulp. A strap band is used to secure the device on the finger proximal phalanx. The working principle of the device is similar to the principle proposed by Minamizawa et al. [18]. When the two motors rotate in opposite directions, the belt is pulled up, providing a force normal to the finger; when the motors spin in the same direction, the belt applies a shear force to the finger. The device weighs $15 \mathrm{~g}$ for $31 \times 28 \times 12 \mathrm{~mm}$ dimensions.

As for the 3-DoF RRS fingertip device, since the servomotors are position controlled, it is only possible to command them with a desired angle. The relationship between the commanded angle and belt displacement for each motor is $\Delta b_{i}=r \Delta \theta_{i}, i=$ 1,2 , where $r=5 \mathrm{~mm}$ is the radius of the servo motor pulley, $\Delta b_{i}$ the commanded belt displacement due to the motion of motor $i$, and $\Delta \theta_{i}$ the $i$-th motor commanded angle expressed in radians. Consider a reference frame $\Sigma_{h}$ placed on the middle of the belt with the $z$-axis pointing toward the finger, the $x$-axis aligned with the belt and the $y$-axis defined accordingly. When the two motors rotate of the same amount $\left(\left|\Delta \theta_{1}\right|=\left|\Delta \theta_{2}\right|\right)$ in opposite directions, the belt is displaced along the $z$-axis of $\Delta b_{i}$. When the two motor rotate of the same amount in the same direction $\Delta \theta_{1}=\Delta \theta_{2}$ the belt is displaced of $\Delta b_{i}$ along the $x$-axis. 


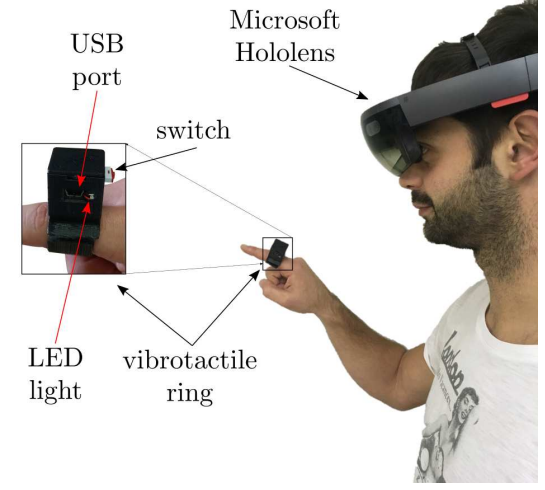

Fig. 2. The vibrotactile ring worn on the proximal phalanx of the index finger is connected to the Microsoft HoloLens headset via BLE. It provides vibration bursts according to the information received from the HoloLens.

To relate the belt displacement to the desired wrench to apply on the finger proximal phalanx $\mathbf{w}_{p, d}$, we assume $\mathbf{w}_{p, d}=$ $[n t]^{T} \in \mathbb{R}^{2}$ as

$$
\mathbf{w}_{p, d}=\left[\begin{array}{c}
n \\
t
\end{array}\right]=\left[\begin{array}{cc}
k_{n} & 0 \\
0 & k_{t}
\end{array}\right]\left[\begin{array}{c}
\Delta \theta_{1} r+\Delta \theta_{2} r \\
\Delta \theta_{1} r-\Delta \theta_{2} r
\end{array}\right],
$$

where $\mathbf{K}=\left[\begin{array}{cc}k_{n} & 0 \\ 0 & k_{t}\end{array}\right]$ is the phalanx stiffness matrix [6].

\section{Wireless vibrotactile ring for the finger}

This device is a novel vibrotactile ring for the proximal finger phalanx and it is shown in Fig. 2. It is composed of an ABS plastic ring, which houses a vibrotactile motor. The motor used is an eccentric rotating mass vibrotactile motor (Precision MicroDrives, United Kingdom). The ring is also equipped with an RFduino, a fingertip-size Arduino-compatible microcontroller (RFduino, USA) that enables Bluetooth Low Energy (BLE) communications with any compatible device, e.g., an external computer or the Microsoft HoloLens. The powering is guaranteed by an embedded $3 \mathrm{~V}$ Lithium Coin Battery, that guarantees long lifetime. A tiny blue LED light is placed on the side of the device's case, to visually show when the vibrotactile motor is active (see the supplementary video). Although other vibrating rings have been presented in [19], [20], this is the first time a similar device is used in an immersive scenario.

\section{Sensory substitution of haptic feedback}

Sensory substitution is the use of one human sense to receive information usually received by another sense. In our case, we want to provide haptic information through a non-haptic feedback modality. In the literature, there are several examples of such sensory substitution approach, especially in the field of robotic surgery [21], [22].

In our experiments, we changed the color of the considered virtual object to provide the user with the corresponding haptic information. For example, if a user touches a virtual object, the color of the object changes according to the amount of force exerted on it, from red to black. A red shade of the object indicates a light grasp, while a black shade indicates a strong grasp. In this condition, no haptic device is worn by the user.

\section{FINGERS TRACKING METHODS}

To achieve compelling and timely haptic feedback, it is paramount to guarantee a reliable and accurate tracking of the fingertips position and orientation. In the first two experiments described in Secs. IV-A and IV-B, the images were registered by an external camera and the tracking was carried out using the ARToolKit 5.3 library. The quality of the tracking is affected by several factors, such as marker shape, sharpness, smoothness, dimension, and positioning. To maximize the quality of our tracking, we followed the design suggestions presented by Khan et al. [23]. To evaluate the performance of this tracking approach, we asked three human users to sit in front of a table and wear visual markers on their thumb and index fingers (as in Fig. 3). Then, we asked them to move freely in 3dimensional space for 5 minutes, moving around four cubes placed on the table. As done in Khan et al. [23], we evaluated (i) the number of true identifications, (ii) the number of false identifications (i.e., inter-marker confusion), and (iii) the confidence factor, which shows the tracking system confidence in the marker identification. We registered an average of 2867 true identifications, 1012 false identifications, and a 0.7602 confidence factor for the true identifications. These results are in agreements with the ones registered in [23] and guaranteed a quite reliable and stable tracking.

In the third experiment of Sec. IV-C, we wanted to focus on a fully-wearable solution. For this reason, we used the Microsoft HoloLens to register the real scene and render the augmented scenario to the user. The gesture recognition API provided by the Microsoft HoloLens was in charge of detecting when the user was performing the "air tap" gesture. So, in this case, users did not have to wear any visual marker. To evaluate the performance of this tracking approach, we asked the same three human users to wear the HoloLens and our vibrotactile ring (as in Fig. 2). Then, we asked them to move freely in 3-dimensional space for 5 minutes, performing the "air tap" gesture as they pleased. The gesture was correctly recognized $91 \%$ of the times.

\section{EXPERIMENTAL EVALUATION}

We carried out three experiments. A video showing all experiments is available as supplemental material.

\section{A. Experiment \#1: box and block}

This experiment is inspired from the "box and block" test, which is a functional test used in upper limb rehabilitation to measure the gross manual dexterity of a patient or of a person using an upper limb prosthesis [24].

1) Setup and Subjects: The experimental setup is composed of a video camera, three (or four) visual markers, a screen, and one object (virtual or real), as shown in Fig. 3. Two markers are worn by the subjects on the thumb and index middle phalanges, one marker is placed on the real object, and one marker is attached on the table supporting the environment. From each marker worn by the subjects, the AR system creates a proxy point, roughly positioned at the fingertip of the corresponding finger. All the interactions between the finger and the virtual objects are mediated by these points. Target positions for both real and virtual objects are represented as transparent volumes. 


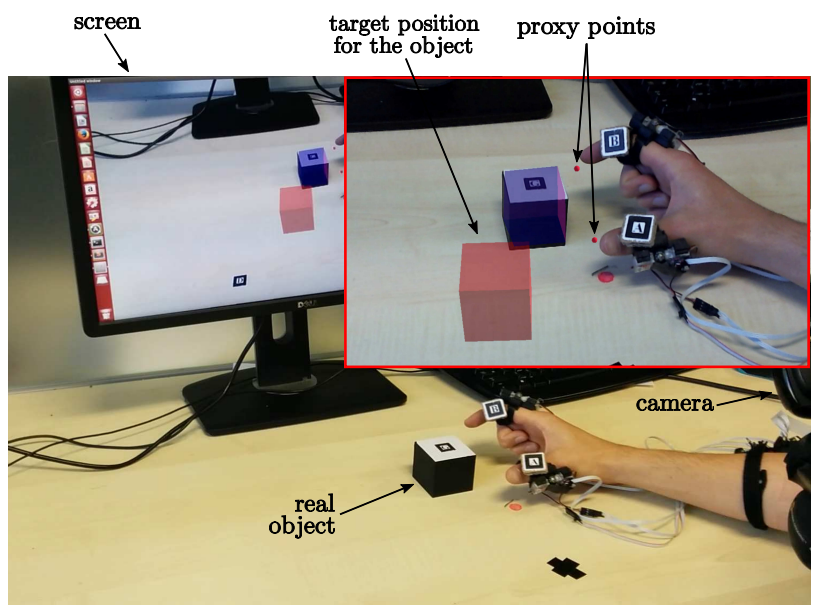

Fig. 3. Experiment \#1: box and block. Subjects move an object (virtual or real) from a random spawning point on the table to a random given target position, indicated by a transparent volume. The task consists of correctly placing as many objects as possible in 60 seconds.

When at least half of the virtual object is inserted into the corresponding volume, the latter turns green to indicate a correct positioning.

Fourteen participants (10 males, 4 females, age range $22-$ 33) took part to the experiment.

2) Methods: The task starts with one object, either virtual or real, placed randomly on the table in front of the subject. The task consists of moving this object to a random target position, indicated by a transparent volume. Once the object is correctly positioned inside its target volume, it is removed from the scene. If the object is virtual, it simply disappears from the scene; if the object is real, it is removed by the experimenter. As soon as the environment is clear, a new object - virtual or real - is again randomly placed on the table. If the new object is virtual, it simply appears on the scene; if the new object is real, it is placed on the table by the experimenter. The subject is then asked again to move the new object to its target volume, and so on. The task consists of correctly placing as many objects as possible in 60 seconds [24].

As the index and thumb fingertips touch the virtual objects, a suitable amount of force $f_{p}$ is provided to the user, according to the feedback modality considered. This interaction force is evaluated according to the god-object model [25]. We modeled the virtual object-fingertip interaction with a spring of stiffness $500 \mathrm{~N} / \mathrm{m}$, a friction coefficient of 0.75 , and a weight of $150 \mathrm{~g}$.

Each participant execute twelve trials of the boxes and blocks task, with three randomized repetitions for each feedback condition proposed:

(CR) haptic feedback provided by the hRing wearable device (see Sec. II-B),

(CF) haptic feedback provided by the 3-DoF wearable device (see Sec. II-A),

(V) sensory substitution via visual feedback (see Sec. II-D), (N) no force feedback.

In condition CR, subjects are required to wear two visual markers and two hRing devices, on the thumb and index fingers (as in Fig. 3). As the user touches a virtual object, the hRing devices provide a suitable amount of cutaneous feedback, as described in Sec. II-B: pressure stimuli to render the stiffness of the object and skin stretch stimuli to render friction/weight

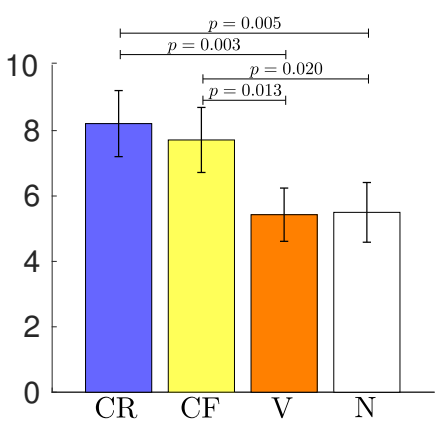

(a) Boxes placed.

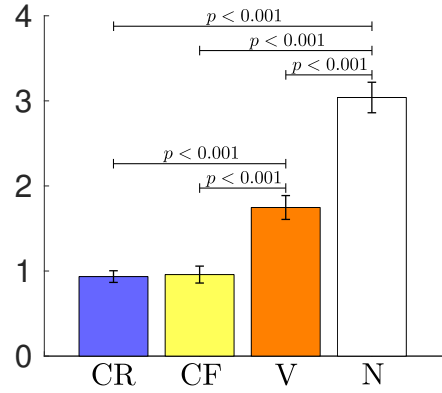

(b) Force (N).

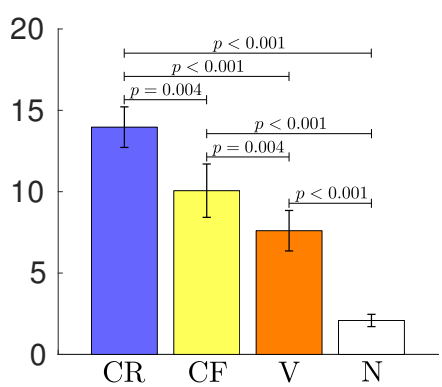

(c) Perceived effectiveness.
Fig. 4. Experiment \#1. Mean and standard deviation of (a) boxes correctly placed, (b) force exerted on the virtual objects, and (c) perceived effectiveness of the four feedback conditions are plotted.

information [18]. In condition $\mathrm{CF}$, subjects are required to wear two visual markers and two 3-RRS devices, on the thumb and index fingers. As the user touches a virtual object, the 3RRS devices provide a suitable amount of cutaneous feedback, as described in Sec. II-A: the platforms move towards the skin to render the stiffness of the object and tilt to render friction/weight information [6]. In condition $\mathrm{V}$, subjects are required to wear two visual markers but no cutaneous devices. As the user touches a virtual object, the color of the object changes according to the amount of force exerted on it, as described in Sec. II-D. A red shade of the object indicates a light grasp, while a black shade indicates a strong grasp. In condition $\mathrm{N}$, subjects are required to wear two visual markers. No devices are worn on the fingers, and no information about the interaction forces are provided to the user. In all conditions, no force feedback is provided when interacting with the real cube.

3) Results: To compare the different metrics, we ran oneway repeated-measures ANOVAs or Friedman tests.

Fig. 4a shows the number of boxes correctly placed in the given time. A Friedman test showed a statistically significant difference between the means of the four feedback conditions $\left(\chi^{2}(3)=22.397, p<0.001, a=0.05\right)$. Post hoc analysis with Bonferroni adjustments revealed a statistically significant difference between CR vs. $\mathrm{N}$ ( $p=0.005), \mathrm{CR}$ vs. V ( $p=0.003)$, CF vs. $\mathrm{N}(p=0.020), \mathrm{CF}$ vs. $\mathrm{V}(p=0.013)$. Fig. $4 \mathrm{~b}$ shows the force exerted by the users on the virtual objects, calculated as the root mean square of $\left\|\mathbf{f}_{\mathbf{p}}\right\|$ (see Sec. IV-A2). The data passed the Shapiro-Wilk normality test. Mauchly's Test of Sphericity indicated that the assumption of sphericity had been violated $\left(\chi^{2}(5)=12.416, p=0.030\right)$. The one-way repeatedmeasure ANOVA with a Greenhouse-Geisser correction revealed statistically significant difference between the feedback 


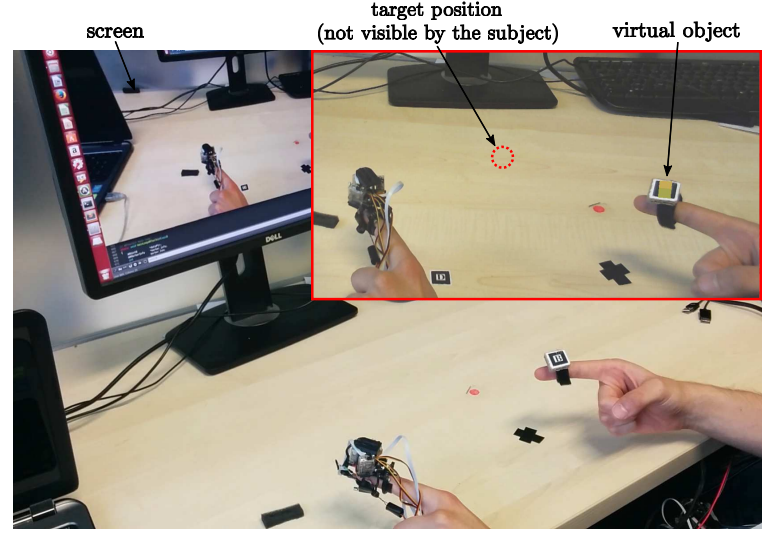

Fig. 5. Experiment \#2: guidance. Subjects need to place the virtual cube in a random target position in the space, which is not visible. This picture shows condition $\mathrm{CF}_{1}$, in which the user wore the 3-DoF wearable device on the contralateral (left) index finger.

conditions $\left(F_{1.802,23.425}=345.555, p<0.001, a=0.05\right)$ Post hoc analysis with Bonferroni adjustments revealed a statistically significant difference between conditions CR vs. V $(p<0.001)$, CR vs. N $(p<0.001)$, CF vs. V $(p<0.001)$, CF vs. $\mathrm{N}(p<0.001)$, $\mathrm{V}$ vs. $\mathrm{N}(p<0.001)$. In addition to the quantitative evaluation reported above, we also measured users' experience. At the end of the experiment, subjects were asked to rate, on a slider going from 0 to 20 , the effectiveness of each feedback condition in completing the given task. Fig. 4c shows the perceived effectiveness for the four feedback conditions. The data passed the Shapiro-Wilk normality test and the Mauchly's Test of Sphericity. The one-way repeated-measure ANOVA revealed statistically significant difference between the feedback conditions $\left(F_{3,39}=81.364, p<0.001, a=0.05\right)$. Post hoc analysis with Bonferroni adjustments revealed a statistically significant difference between all conditions: $\mathrm{CR}$ vs. CF $(p=0.004)$, CR vs. V $(p<0.001)$, CR vs. $\mathrm{N}(p<0.001)$, $\mathrm{CF}$ vs. $\mathrm{N}(p<0.001), \mathrm{CF}$ vs. $\mathrm{V}(p=0.004), \mathrm{V}$ vs. $\mathrm{N}$ $(p<0.001)$. Finally, we asked which of the two considered wearable haptic systems was more unobtrusive. Thirteen out of fourteen subjects chose the hRing.

\section{B. Experiment \#2: guidance}

This second experiment considers a guidance task, which is particularly relevant in industrial training applications. The trainees can directly look at the console/machine they need to command, while the AR and wearable haptic systems provide them with specific and unobtrusive information about the task to learn (e.g., how to correctly place some material inside a broaching machine).

1) Setup and Subjects: The experimental setup is composed of a video camera, two visual markers, and a screen, as shown in Fig. 5. One marker is worn by the subjects on the right index middle phalanx and one marker is attached on the table supporting the environment. From the marker worn by the subjects, the AR system creates a cube (yellow object in Fig. 5), roughly positioned at the finger middle phalanx.

Ten participants (10 males, age range $23-33)$ took part to the experiment.

2) Methods: The task consisted of placing the cube rendered on the subject's index finger in a target position, as precisely and

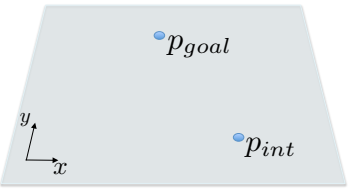

(a)

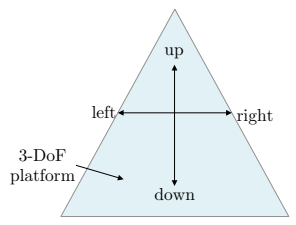

(c)

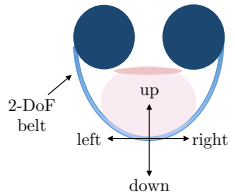

(b)

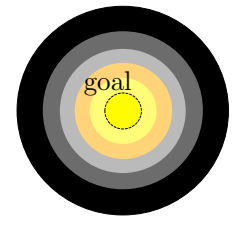

(d)
Fig. 6. Experiment \#2: guidance. In (a) the proposed scenario. In (b) and (c), how the 2-DoF and the 3-DoF devices suggest the directions. In (d), the principle used for sensory substitution: the color of the cube changes from black to yellow according to the distance to the goal.

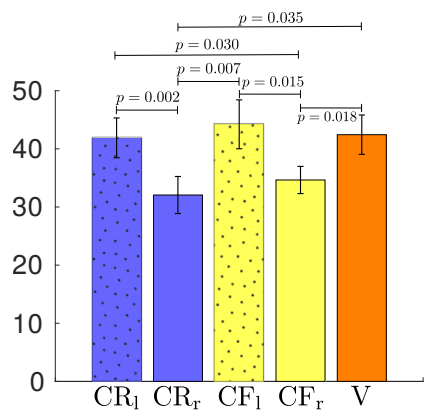

(a) Time (s).

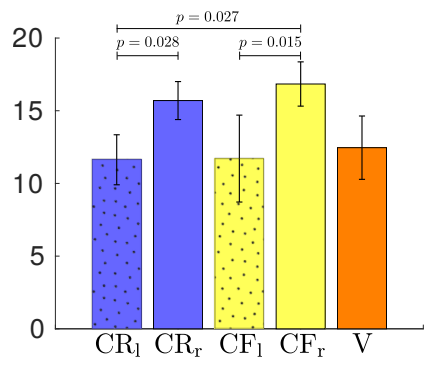

(b) Perceived effectiveness.

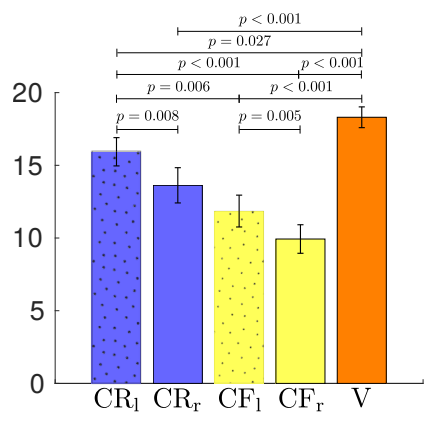

(c) Perceived wearability.

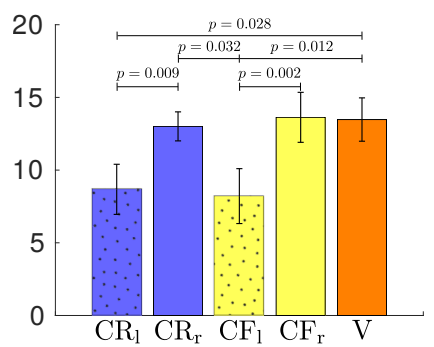

(d) Perceived intuitiveness.
Fig. 7. Experiment \#2. Mean and standard deviation of (a) task completion time, (b) perceived effectiveness, (c) wearability, and (d) intuitiveness of the five feedback conditions are plotted.

as fast as possible. Differently from the previous experiment, the target position was not visible on the screen, and subjects had to solely rely on the haptic guidance information provided by the system, according to the feedback modality considered.

Each participant carries out nine trials of the guidance task, with three randomized repetitions for each feedback condition:

(CR) guidance haptic cues provided by the hRing device,

(CF) guidance haptic cues provided by the 3-DoF device,

(V) sensory substitution via visual feedback.

In Fig. 6 we show how the guidance is obtained in the three conditions. We consider the planar case, where the height of the hand with respect to the table is not taken into account. In other words, the user is guided toward a certain goal position $p_{\text {goal }} \in \mathbb{R}^{2}$ starting from an initial position $p_{\text {int }} \in \mathbb{R}^{2}$ defined 
w.r.t. the marker placed on the table, see Fig. 6a. In condition $\mathrm{CR}$, once the hRing is worn, the belt is placed at the middle of the possible displacement along the $z$-axis (see Sec. II-B). From there, the displacement of the belt is generated proportionally to the position error computed as $p_{\text {err }}=p_{\text {goal }}-p_{\text {int }}$. In particular, the error along the $y$-axis is mapped onto a displacement along the normal to the finger pad, whereas the error along the $x$-axis is mapped onto a shear displacement, see Fig. $6 \mathrm{~b}$. In condition $(\mathrm{CF})$, subjects are required to wear one 3-RRS devices. Similarly to the previous condition, the position error is mapped onto platform rotations as reported in Fig. 6c: when the fingertip is pushed on the right side tilting the platform it means the user has to move to the right, and so on. Finally, in condition V, subjects do not wear any wearable haptic device. The color of the object changes according to the distance from the target position, from yellow to black. A yellow shade of the object indicates that the target position is very near, while a black shade indicates that the target position is still far, see Fig. 6d. We tested conditions CR and CF with the devices worn either on the left index finger (controlateral to the finger with the visual marker, conditions $\mathrm{CR}_{1}$ and $\mathrm{CF}_{1}$ ) or on the right index finger (on the same finger with the visual marker, conditions $\mathrm{CR}_{\mathrm{r}}$ and $\mathrm{CF}_{\mathrm{r}}$ ). Considering the three feedback conditions and the two positions for the wearable devices, we ended up with five different experimental modalities.

3) Results: All subjects succeeded in placing the virtual object within $1 \mathrm{~cm}$ from the target location in less than 1 minute in all conditions.

Fig. 7a shows the task completion time. The data passed the Shapiro-Wilk normality test and the Mauchly's Test of Sphericity. The one-way repeated-measure ANOVA revealed statistically significant difference between the feedback conditions $\left(F_{4,36}=10.675, p<0.001, a=0.05\right)$. Post hoc analysis with Bonferroni adjustments revealed a statistically significant difference between conditions $\mathrm{CR}_{\mathrm{l}}$ vs. $\mathrm{CR}_{\mathrm{r}}(p=0.002), \mathrm{CR}_{\mathrm{l}}$ vs. $\mathrm{CF}_{\mathrm{r}}(p=0.030), \mathrm{CR}_{\mathrm{r}}$ vs. $\mathrm{CF}_{\mathrm{l}}(p=0.007), \mathrm{CR}_{\mathrm{r}}$ vs. $\mathrm{V}(p=0.035), \mathrm{CF}_{\mathrm{l}}$ vs. $\mathrm{CF}_{\mathrm{r}}(p=0.015)$, and $\mathrm{CF}_{\mathrm{r}}$ vs. V $(p=0.018)$.

After the experiment, subjects were asked to rate (i) the effectiveness of the guidance system, (ii) its wearability, and (iii) its intuitiveness on a slider going from 0 to 20. Fig. $7 \mathrm{~b}$ shows the perceived effectiveness. The data passed the Shapiro-Wilk normality test and the Mauchly's Test of Sphericity. The one-way repeated-measure ANOVA revealed statistically significant difference between the feedback conditions $\left(F_{4,36}=5.806, p=0.001, a=0.05\right)$. Post hoc analysis with Bonferroni adjustments revealed a statistically significant difference between conditions $\mathrm{CR}_{\mathrm{l}}$ vs. $\mathrm{CR}_{\mathrm{r}}(p=0.028)$, $\mathrm{CR}_{\mathrm{l}}$ vs. $\mathrm{CF}_{\mathrm{r}}(p=0.027)$, and $\mathrm{CF}_{\mathrm{l}}$ vs. $\mathrm{CF}_{\mathrm{r}}(p=0.015)$. Fig. 7c shows the perceived wearability. The data passed the Shapiro-Wilk normality test and the Mauchly's Test of Sphericity. The one-way repeated-measure ANOVA revealed statistically significant difference between the feedback conditions $\left(F_{4,36}=39.577, p<0.001, a=0.05\right)$. Post hoc analysis with Bonferroni adjustments revealed a statistically significant difference between $\mathrm{CR}_{\mathrm{l}}$ vs. $\mathrm{CR}_{\mathrm{r}}(p=0.008)$, $\mathrm{CR}_{\mathrm{l}}$ vs. $\mathrm{CF}_{\mathrm{l}}(p=0.006), \mathrm{CR}_{\mathrm{l}}$ vs. $\mathrm{CF}_{\mathrm{r}}(p<0.001), \mathrm{CR}_{\mathrm{l}}$ vs. $\mathrm{V}(p=0.027), \mathrm{CR}_{\mathrm{r}}$ vs. $\mathrm{V}(p<0.001), \mathrm{CF}_{\mathrm{l}}$ vs. $\mathrm{CF}_{\mathrm{r}}$ $(p=0.005), \mathrm{CF}_{1}$ vs. $\mathrm{V}(p<0.001)$, and $\mathrm{CF}_{\mathrm{r}}$ vs. $\mathrm{V}$ $(p<0.001)$. Fig. 7d shows the perceived intuitiveness. The data passed the Shapiro-Wilk normality test and the Mauchly's Test of Sphericity. The one-way repeated-measure ANOVA revealed statistically significant difference between the feedback conditions $\left(F_{4,36}=11.288, p<0.001, a=0.05\right)$. Post hoc analysis with Bonferroni adjustments revealed a statistically significant difference between $\mathrm{CR}_{\mathrm{l}}$ vs. $\mathrm{CR}_{\mathrm{r}}(p=0.009), \mathrm{CR}_{\mathrm{l}}$ vs. $\mathrm{V}(p=0.028), \mathrm{CR}_{\mathrm{r}}$ vs. $\mathrm{CF}_{\mathrm{l}}(p=0.032), \mathrm{CF}_{\mathrm{l}}$ vs. $\mathrm{CF}_{\mathrm{r}}$ $(p=0.002)$, and $\mathrm{CF}_{1}$ vs. $\mathrm{V}(p=0.012)$.

\section{Experiment \#3: gaming}

While the first two experiments employed an external camera to capture the real environment and an LCD screen to show the augmented scenario to the user, in this third experiment we wanted to employ a fully-wearable and portable solution. The external camera and LCD screen are substituted by the Microsoft HoloLens, which seamlessly superimposes the virtual scene onto the real environment. To evaluate wearable haptics in this new context, we considered a gaming experience. The gaming business is powering an increasing amount of money, but the haptic feedback provided by commercially-available gaming solutions is still very limited and rudimentary [1].

1) Setup and Subjects: The experimental setup is composed of a Microsoft HoloLens and the vibrotactile ring, as shown in Fig. 2. Ten participants ( 8 males, 2 females, age range $23-$ 34) took part to the experiment.

2) Methods: The task consisted of playing the mixed reality first-person shooter RoboRaid game for 5 minutes in endless mode. The game consists of defending your home against a (virtual) robot/alien invasion. Users can aim the weapon via gaze and fire using the "air tap" gesture, which consists of holding the hand straight out in a loose fist with the index finger straight up toward the ceiling, tapping your finger down and then quickly raising it back up again. We started recording the time as soon as the subject was able to shoot (we skipped the introductory part). Whether a "game over" occurs within the 5 minutes, we stop the stopwatch, restart the game session, and then restart the stopwatch again (without reset), until reaching the predefined playing time.

Each participant carries out two sessions of the RoboRaid task, one for each feedback condition proposed:

(CV) gesture haptic feedback provided by the wearable vibrotactile ring (see Sec. II-C),

(N) no haptic feedback.

In condition $\mathrm{CV}$, subjects are required to wear one wearable vibrotactile ring on the right index finger and play the game. Every time the HoloLens recognizes the "air tap" gesture, it makes the weapon fire and the vibrotactile ring provides a 100 -ms-long vibration burst. In condition $\mathrm{N}$, subjects do not wear the wearable vibrotactile ring and do not receive any haptic feedback when shooting the weapon.

3) Results: We evaluated the number of robots destroyed during the game session in the two conditions. The data passed the Shapiro-Wilk normality test. A paired-samples t-test determined that the number of robots destroyed did not differed statistically significantly between the two conditions $(\mathrm{CV}$ : $66.9 \pm 7.4 ; \mathrm{N}: 64.7 \pm 7.0$ robots, mean \pm std. deviation). Moreover, we evaluated the immersiveness of the haptic-enabled virtual 
TABLE II

EXPERIMENT \#3. USEFULNESS, SATISFACTION, AND EASE OF USE (USE) QUESTIONNAIRE RESULTS (STAT. DIFFERENT VALUES IN RED).

\begin{tabular}{|lll|}
\hline QA. & Usefulness (condition CV with respect to condition N) & \\
\hline 1. & It helps me be more effective. & $7.2 \pm 0.78$ \\
2. & It helps me be more productive. & $7.0 \pm 0.94$ \\
3. & It is useful. & $7.8 \pm 0.63$ \\
4. & It makes the things I want to accomplish easier to get done. & $6.7 \pm 0.67$ \\
\hline
\end{tabular}

\begin{tabular}{|llcc|}
\hline QB. & Ease of use & $\mathrm{CV}$ & $\mathrm{N}$ \\
\hline 1. It is easy to use. & $8.2 \pm 0.78$ & $8.1 \pm 0.73$ \\
2. $\quad$ It is user friendly. & $7.7 \pm 0.67$ & $7.9 \pm 0.73$ \\
3. $\quad$ Using it is effortless. & $7.2 \pm 0.79$ & $7.9 \pm 0.74$ \\
4. $\quad$ I can use it without written instructions. & $7.2 \pm 0.78$ & $7.1 \pm 0.73$ \\
5. I don't notice any inconsistencies as I use it. & $7.1 \pm 0.73$ & $7.7 \pm 0.67$ \\
6. $\quad$ Both occasional and regular users would like it. & $8.1 \pm 0.73$ & $7.9 \pm 0.73$ \\
7. $\quad$ I can use it successfully every time. & $7.4 \pm 0.69$ & $7.6 \pm 0.51$ \\
\hline QC. Ease of learning & $\mathrm{CV}$ & $\mathrm{N}$ \\
\hline 1. I learned to use it quickly. & $8.0 \pm 0.94$ & $8.2 \pm 0.78$ \\
2. It is easy to learn to use it. & $7.9 \pm 0.73$ & $8.0 \pm 0.66$ \\
3. $\quad$ I quickly became skillful with it. & $8.2 \pm 0.78$ & $8.0 \pm 0.81$ \\
\hline QD. Satisfaction & $\mathrm{CV}$ & $\mathrm{N}$ \\
\hline 1. I am satisfied with it. & $8.1 \pm 0.74$ & $7.3 \pm 0.67$ \\
2. It is fun to use. & $7.8 \pm 0.63$ & $7.0 \pm 0.67$ \\
3. It works the way I want it to work. & $7.5 \pm 1.08$ & $7.3 \pm 0.67$ \\
4. It is wonderful. & $8.3 \pm 0.67$ & $7.2 \pm 0.79$ \\
5. It is pleasant to use. & $7.3 \pm 0.82$ & $7.3 \pm 0.67$ \\
\hline
\end{tabular}

reality scenario through the Usefulness, Satisfaction, and Ease of use (USE) questionnaire [26], asking subjects to compare the experience while wearing the wearable vibrotactile ring vs. bare hand. Our USE questionnaire is composed of 21 Likerttype questions. It contained a set of assertions, where a score of 9 was described as "completely agree" and a score of 1 as "completely disagree" with the assertion. Questions and results are reported in Table II. The first set of questions (QA in Table II) considered the usefulness of having the wearable vibrotactile devices $(\mathrm{CV})$ with respect to not having any haptic feedback $(\mathrm{N})$. The other three sets of questions $(\mathrm{QB}, \mathrm{QC}$, and QD in Table II) were asked separately for both conditions. In order to determine whether the data registered in sets B, C, and $\mathrm{D}$ differ between the two feedback conditions, we ran seventeen Wilcoxon signed-rank tests (significance level alpha $=0.05)$, one for each question. The analysis revealed significant statistical difference between answers QB6 $(p=0.034)$, QD1 $(\mathrm{p}=0.035), \mathrm{QD} 2(\mathrm{p}=0.011)$, and $\mathrm{QD} 4(\mathrm{p}=0.031)$. Answers that resulted statistically significantly different are reported in red in Table II.

We also asked which condition the subjects preferred. All subjects preferred the condition where they were wearing the wearable vibrotactile ring (condition $\mathrm{CV}$ ).

\section{Discussion And Conclusion}

In this paper we studied and evaluated how wearable finger haptics can enhance user experience in Augmented Reality. Sec. IV-A presents the first experiment, considering a manipulation task where subjects had to re-locate as many objects as possible in a given time frame. We compared task's performance when providing the user with haptic feedback through the hRing, haptic feedback through the 3-DoF device, sensory substitution via visual cues, and no haptic feedback at all. Results showed that providing haptic feedback through the wearable devices significantly improved the performance of the considered task, in terms of number of placed objects, force, and perceived effectiveness. We chose the interaction force as a measurement of the device transparency, because less force applied to the virtual object means that users have promptly stopped their fingers when in contact with the object. This latter metric is also useful to understand how well the display of virtual stiffness works: if users keep moving their fingers after contact, the virtual object may indeed be perceived as softer than it really is. It is interesting to also highlight that we gave no explicit indication regarding the amount of force to apply when grasping an object. The fact that users anyway applied less force during the haptics conditions shows that wearable haptic feedback is able to elicit a natural and compelling feeling of contact. We registered a difference between the two wearable devices in the perceived effectiveness metric only. In fact, subjects found the hRing more effective and, in general, more "wearable". This result was expected since the hRing is significantly smaller and lighter and its position at the proximal finger phalanx enables a direct contact with the real environment. The capability of the hRing to leave the fingertip free to interact with real objects was indeed well appreciated by the subjects, confirming the results of [12]. On the other hand, the rigid platform of the 3-DoF device severely impaired the users when they had to interact with the real environment, since it remained between the fingertip and the object to grasp. However, the hRing has only 2-DoF and therefore it cannot provide the same information as the 3-DoF fingertip device. For this reason, if we need to provide richer information, the 3-DoF fingertip device may still be a better choice.

The second experiment of Sec. IV-B considers a guidance task, where subjects had to follow haptic cues to place a virtual object. We compared task's performance when providing the user with haptic cues through the hRing, haptic cues through the 3-DoF device, and sensory substitution via visual cues. We also compared conditions while wearing the wearable devices either on the right or left index finger. Results showed again that providing haptic feedback through the wearable haptic devices significantly improved the performance of the considered task, in terms of completion time, and perceived effectiveness, wearability, and intuitiveness. Moreover, results improved when haptic feedback was provided on the same hand which moved the virtual object (i.e., the right hand). Sensory substitution via visual feedback also showed a rather high performance. This result is quite surprising considering that sensory substitution only provided 1-D information about the distance from the target position, while the haptic conditions conveyed higher-dimensional information. In the future, we plan to test this experiment while also providing 2-D visual feedback.

In the first two experiments, an external camera was in charge of capturing the real environment, the augmented scene was shown on a LCD screen, and the wearable haptic devices were wired to an external unit in charge of providing power and communication with the computer. While this setup is acceptable for a proof of concept and first evaluation, it is unsuitable to demonstrate the applicability, effectiveness, and 
readiness of wearable haptics for use in everyday life and the entertainment industry. The use of this non-wearable setup was also the main limitation of the work presented by Maisto et al. [12]. For this reason, in this work we also considered a third experiment which explores a simpler, but fully-wearable and portable solution. The external camera and LCD screen were substituted by the Microsoft HoloLens, which seamlessly superimposed the virtual scene onto the real environment. To evaluate wearable haptics in this new context, we considered a gaming task, in which users had to play a first-person shooter game and fire their weapon using the "air tap" HoloLens gesture. We compared user performance and experience when providing haptic feedback through a lightweight wireless vibrotactile ring vs. no haptic feedback at all. While the presence of haptics did not affect the player performance, results showed that providing haptic feedback through the wearable haptic device significantly improved the immersiveness and satisfaction of the task. Moreover, all subjects greatly preferred using the vibrotactile ring and rated it as very wearable and comfortable. These results prove that we can improve the immersiveness of AR systems even with very simple, market-ready devices such as our vibrotactile ring, taking advantage of their extreme wearability, comfort, and ease of use. Indeed, haptics seems to play a primary importance role in improving one of the major factor for videogames: the immersiveness. Of course, more complex devices are expected to enable richer interactions. A final important consideration is the price tag on our system. The vibrotactile ring, hRing, and 3-DoF devices can be assembled with less the one hundred dollars, making our method especially attractive for all those applications where grounded kinesthetic interfaces are too expensive or too complicated to use (e.g., gaming, at-home rehabilitation).

In the next future, we will extend this evaluation to include even more wearable devices, able to apply different cutaneous stimuli to different part of the body. Specifically, we will study more in detail the effect of vibrotactile stimuli in AR, and we will consider haptic devices for the wrist and arm (e.g., [27], [28]). Finally, we intend to better investigate the role of sensory substitution of visual feedback, combining visual and haptic information together to achieve higher performance.

\section{REFERENCES}

[1] C. Pacchierotti, S. Sinclair, M. Solazzi, A. Frisoli, V. Hayward, and D. Prattichizzo, "Wearable haptic systems for the fingertip and the hand Taxonomy, review, and perspectives," IEEE Trans. Haptics, vol. 10, no. 4 pp. 580-600, 2017.

[2] A. Frisoli, M. Solazzi, F. Salsedo, and M. Bergamasco, "A fingertip haptic display for improving curvature discrimination," Presence: Teleoperators Virtual Environ, vol. 17, no. 6, pp. 550-561, 2008.

[3] M. Gabardi, M. Solazzi, D. Leonardis, and A. Frisoli, "A new wearable fingertip haptic interface for the rendering of virtual shapes and surface features," in Proc. IEEE Haptics Symposium, 2016, pp. 140-146.

[4] D. Prattichizzo, F. Chinello, C. Pacchierotti, and M. Malvezzi, "Towards wearability in fingertip haptics: A 3-DoF wearable device for cutaneous force feedback," IEEE Trans. Haptics, vol. 6, no. 4, pp. 506-516, 2013

[5] F. Chinello, M. Malvezzi, C. Pacchierotti, and D. Prattichizzo, "Design and development of a 3RRS wearable fingertip cutaneous device," in Proc. IEEE Int. Conf. Adv. Intelligent Mechatronics, 2015, pp. 293-298.

[6] F. Chinello, C. Pacchierotti, M. Malvezzi, and D. Prattichizzo, "A three revolute-revolute-spherical wearable fingertip cutaneous device for stiffness rendering," IEEE Trans. Haptics, vol. 11, no. 1, pp. 39-50, 2018.
[7] D. Leonardis, M. Solazzi, I. Bortone, and A. Frisoli, "A 3-RSR haptic wearable device for rendering fingertip contact forces," IEEE Trans. Haptics, vol. 10, no. 3, pp. 305-316, 2017.

[8] A. Girard, M. Marchal, F. Gosselin, A. Chabrier, F. Louveau, and A. Lécuyer, "Haptip: Displaying haptic shear forces at the fingertips for multi-finger interaction in virtual environments," Frontiers in ICT, vol. 3, p. 6, 2016.

[9] S. B. Schorr and A. M. Okamura, "Three-dimensional skin deformation as force substitution: Wearable device design and performance during haptic exploration of virtual environments," IEEE Trans. Haptics, vol. 10, no. 3, pp. 418-430, 2017.

[10] C. Pacchierotti, G. Salvietti, I. Hussain, L. Meli, and D. Prattichizzo, "The hRing: A wearable haptic device to avoid occlusions in hand tracking," in Proc. IEEE Haptics Symposium, 2016, pp. 134-139.

[11] S. Scheggi, G. Salvietti, and D. Prattichizzo, "Shape and weight rendering for haptic augmented reality," in Proc. IEEE Int. Symp. Robots and Human Interactive Communications, 2010, pp. 44-49.

[12] M. Maisto, C. Pacchierotti, F. Chinello, G. Salvietti, A. De Luca, and D. Prattichizzo, "Evaluation of wearable haptic systems for the fingers in augmented reality applications," IEEE Trans. Haptics, vol. 10, no. 4, pp. 511-522, 2017.

[13] X. De Tinguy, C. Pacchierotti, M. Marchal, and A. Lecuyer, "Enhancing the stiffness perception of tangible objects in mixed reality using wearable haptics," in Proc. IEEE Virtual Reality, 2018.

[14] O. Bau and I. Poupyrev, "REVEL: Tactile feedback technology for augmented reality," ACM Trans. Graphics, vol. 31, no. 4, p. 89, 2012.

[15] S. L. Joseph, X. Zhang, I. Dryanovski, J. Xiao, C. Yi, and Y. Tian, "Semantic indoor navigation with a blind-user oriented augmented reality," in Proc. IEEE Int. Conf. Systems, Man, and Cybernetics (SMC), 2013, pp. 3585-3591.

[16] V. Buchmann, M. Billinghurst, and A. Cockburn, "Directional interfaces for wearable augmented reality," in Proc. Int. Conf. Human-Computer Interaction, 2008, pp. 47-54.

[17] D. Tsetserukou, K. Sato, and S. Tachi, "ExoInterfaces: Novel exosceleton haptic interfaces for virtual reality, augmented sport and rehabilitation," in Proc. 1st Augmented Human Int. Conf., 2010, pp. 1:1-1:6.

[18] K. Minamizawa, S. Fukamachi, H. Kajimoto, N. Kawakami, and S. Tachi, "Gravity grabber: wearable haptic display to present virtual mass sensation," in Proc. ACM SIGGRAPH emerging tech., 2007, p. 8.

[19] O. Ariza, P. Lubos, F. Steinicke, and G. Bruder, "Ring-shaped haptic device with vibrotactile feedback patterns to support natural spatial interaction," in Proc. Eurographics Symposium on Virtual Environments, 2015, pp. 175-181.

[20] E. Freeman, S. Brewster, and V. Lantz, "Tactile feedback for abovedevice gesture interfaces: adding touch to touchless interactions," in Proc. 16th International Conference on Multimodal Interaction. ACM, 2014, pp. $419-426$.

[21] M. Kitagawa, D. Dokko, A. M. Okamura, and D. D. Yuh, "Effect of sensory substitution on suture-manipulation forces for robotic surgical systems," Journal of Thoracic and Cardiovascular Surgery, vol. 129, no. 1, pp. 151-158, 2005.

[22] J. C. Gwilliam, M. Mahvash, B. Vagvolgyi, A. Vacharat, D. D. Yuh, and A. M. Okamura, "Effects of haptic and graphical force feedback on teleoperated palpation," in Proc. IEEE Int. Conf. Robotics and Automation, 2009, pp. 677-682.

[23] D. Khan, S. Ullah, and I. Rabbi, "Factors affecting the design and tracking of artoolkit markers," Computer Standards \& Interfaces, vol. 41, pp. 56-66, 2015.

[24] V. Mathiowetz, G. Volland, N. Kashman, and K. Weber, "Adult norms for the box and block test of manual dexterity," American Journal of Occupational Therapy, vol. 39, no. 6, pp. 386-391, 1985.

[25] C. B. Zilles and J. K. Salisbury, "A constraint-based god-object method for haptic display," in Proc. IEEE/RSJ Int. Conf. Intelligent Robots and Systems, vol. 3, 1995, pp. 146-151.

[26] A. M. Lund, "Measuring usability with the use questionnaire," Usability interface, vol. 8, no. 2, pp. 3-6, 2001.

[27] M. Aggravi, F. Paus, P. Robuffo Giordano, and C. Pacchierotti, "Design and evaluation of a wearable haptic device for skin stretch, pressure, and vibrotactile stimuli," IEEE Robotics and Automation Letters, vol. 3, no. 3, pp. 2166-2173, 2018.

[28] F. Chinello, C. Pacchierotti, J. Bimbo, N. G. Tsagarakis, and D. Prattichizzo, "Design and evaluation of a wearable skin stretch device for haptic guidance," IEEE Robotics and Automation Letters, vol. 3, no. 1, pp. 524-531, 2018. 